\title{
A formação do enfermeiro para o cuidado ao idoso: uma análise documental do ensino de graduação no Paraná
}

RESUMO | Objetivo: Analisar a inserção do cuidado ao idoso nos cursos de graduação em enfermagem das Instituições de Ensino Superior (IES) públicas e privadas do Paraná. Método: Trata-se de estudo descritivo, exploratório, de abordagem qualitativa, que utilizou a análise documental. Foi realizada a identificação das IES e cursos de graduação por meio do acesso à plataforma online do Ministério da Educação e, posteriormente, às páginas oficiais das IES para identificar conteúdos relacionados ao cuidado gerontológico. As ementas foram analisadas pelo Software Interface R IraMuTeQ® Resultados: Foram identificadas 60 IES e obteve-se informações de 52 cursos. Destes, 53,9\% ofertavam disciplinas específicas de saúde do idoso e 46,1\% possuíam disciplinas mistas. Na análise de similitude os principais eixos organizadores foram: idoso, enfermagem e processo. Conclusão: Pouco mais da metade dos cursos de enfermagem aborda o cuidado ao idoso em disciplinas específicas. A formação deve estar alinhada às políticas da atenção à pessoa idosa.

Descritores: Idoso; Saúde do Idoso; Enfermagem Geriátrica; Programas de Graduação em Enfermagem; Educação Superior.

ABSTRACT | Objective: To analyze the insertion of elderly care in undergraduate nursing courses at public and private Higher Education Institutions (HEls) in Paraná. Method: This is a descriptive, exploratory study, with a qualitative approach, which used document analysis. The identification of HEls and undergraduate courses was carried out through access to the Ministry of Education's online platform and, later, to the official pages of the HEls to identify content related to gerontological care. The menus were analyzed by the R IraMuTeQ® Interface Software. Results: $60 \mathrm{HEls}$ were identified and information was obtained from 52 courses. Of these, $53.9 \%$ offered specific subjects on elderly health and $46.1 \%$ had mixed subjects. In the similarity analysis, the main organizing axes were: elderly, nursing and process. Conclusion: Just over half of nursing courses address elderly care in specific disciplines. Training must be aligned with care policies for the elderly.

Keywords: Elderly; Elderly Health; Geriatric Nursing; Undergraduate Nursing Programs; Higher education.

RESUMEN | Objetivo: Analizar la inserción del cuidado de ancianos en los cursos de graduación en enfermería de Instituciones de Enseñanza Superior (IES) públicas y privadas de Paraná. Método: Se trata de un estudio descriptivo, exploratorio, con abordaje cualitativo, que utilizó el análisis de documentos. La identificación de las IES y carreras de grado se realizó a través del acceso a la plataforma en línea del Ministerio de Educación y, posteriormente, a las páginas oficiales de las IES para identificar contenidos relacionados con la atención gerontológica. Los menús fueron analizados por el software de interfaz R IraMuTeQ®. Resultados: Se identificaron 60 IES y se obtuvo información de 52 cursos. De estos, 53,9\% ofrecieron temas específicos sobre la salud del anciano y $46,1 \%$ temas mixtos. En el análisis de similitud, los principales ejes organizadores fueron: anciano, enfermería y proceso. Conclusión: poco más de la mitad de los cursos de enfermería abordan el cuidado de ancianos en disciplinas específicas. La formación debe estar alineada con las políticas de atención a las personas mayores.

Palabras claves: Anciano; Salud del Anciano; Enfermería Geriátrica; Programas de Pregrado en Enfermería; Educación más alta.

\section{Ana Clara Ruocco Vargas}

Enfermeira pela Universidade Estadual de Maringá. Residente em Pneumologia HSP - Unifesp.

ORCID: 0000-0002-5630-6303

\section{Flávia Maria Derhun}

Enfermeira. Doutora em Enfermagem. Universidade Estadual de Maringá. Enfermeira na Seção de Atenção Primária da $15^{\mathrm{a}}$ Regional da Saúde da Secretaria de Estado da Saúde do Paraná.

ORCID: 0000-0003-2653-5022

\section{Luiz Hiroshi Inoue}

Enfermeiro. Universidade Estadual de Maringá. Mestrando pelo Programa de Pós-Graduação em Enfermagem da Universidade Estadual de Maringá

ORCID: 0000-0002-7226-9661

\section{Vanessa Denardi Antoniassi Baldissera}

Enfermeira. Doutora em Enfermagem. Universidade Estadual de Maringá. Docente do Departamento de Enfermagem e do Programa de Pós-graduação em enfermagem na Universidade Estadual de Maringá. ORCID: 0000-0003-1680-9165

\section{Maria Aparecida Salci}

Enfermeira. Doutora em Enfermagem. Universidade Estadual de Maringá. Docente do Departamento de Enfermagem e do Programa de Pós-graduação em enfermagem na Universidade Estadual de Maringá ORCID: 0000-0002-6386-1962

\section{Lígia Carreira}

Enfermeira. Pós-doutora em Enfermagem. Universidade Estadual de Maringá. Docen- te do Departamento de Enfermagem e do Programa de Pós-graduação em enfermagem na Universidade Estadual de Maringá ORCID: 0000-0003-3891-4222

Recebido em: 17/10/2021

Aprovado em: 07/01/2022

\section{INTRODUÇÃO}

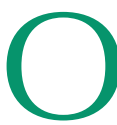
Brasil passa por relevantes mudanças no perfil demográfico, com significativo crescimento do contingente de idosos em detrimento das faixas etárias mais jovens.(1) Este panorama apresenta um desafio inédito e demanda políticas capazes de lidar com 
a heterogeneidade do envelhecimento para que os indivíduos vivam, pelo maior tempo possível, de forma autônoma e independente.(2)

A Política Nacional de Saúde da Pessoa Idosa propõe ações cujo objetivo é manter e/ou promover a capacidade funcional das pessoas com mais de 60 anos. Uma destas é o reajuste na grade curricular dos cursos das Instituições de Ensino Superior (IES), para que consigam abranger os aspectos do envelhecimento e saúde do idoso de forma mais ampla.(3) Anteriormente a esta, a Política Nacional do Idoso já pontuava a necessidade da inclusão da Geriatria e Gerontologia como disciplina dos cursos de graduação da área da saúde(4) e o Estatuto do Idoso sobre conteúdos voltados ao processo de envelhecimento a todos os níveis de ensino.(5)

As Diretrizes Curriculares Nacionais (DCN) criadas para orientar a concepção dos currículos das IES do Brasil, buscam nos cursos de saúde, uma formação de profissionais que reconheçam as demandas para que possam intervir oportunamente(6) No que tange à pessoa idosa, o enfermeiro, em especial, deve possuir competências para atuar na atenção à saúde,(6-7) bem como na elaboração e execução de políticas públicas,(8) em todos os níveis de atenção que atendem o referido público. Para tanto, é necessário que o enfermeiro possua em sua formação conteúdos que contemplem a saúde do idoso em sua multidimensionalidade.

Ao considerar que o ensino sobre a saúde do idoso na graduação em enfermagem influencia na qualidade do cuidado e que não há uniformidade deste entre os cursos ofertados, bem como do desenvolvimento de competências para o atendimento do público idoso(9) torna-se inevitável o questionamento: como a temática cuidado ao idoso está inserida nos cursos de graduação em enfermagem do estado do Paraná?
OBJETIVO

Analisar a inserção do cuidado ao idoso nos cursos de graduação em enfermagem das IES públicas e privadas do Paraná.

\section{MÉTODO}

Trata-se de um estudo descritivo, exploratório, de abordagem qualitativa, utilizando-se da análise documental das grades curriculares e ementas dos cursos de graduação em enfermagem de IES paranaenses.

Em dezembro de 2018 foi realizada a identificação das IES e cursos de graduação, por meio do acesso à plataforma online do Ministério da Educação (e-MEC). Entre dezembro de 2018 e janeiro de 2019, foram acessadas as páginas oficiais das IES para identificar de que forma a temática cuidado ao idoso estava inserida. Para tanto, buscou-se nas grades curriculares e ementas a existência de uma disciplina específica de saúde do idoso ou se o cuidado a este era abordado em outra disciplina, não específica.

Nos casos em que as informações buscadas não constavam no site da IES foi encaminhado e-mail aos coordenadores solicitando-as. Após 30 dias foi realizado contato via telefone com as IES que não disponibilizaram as informações via e-mail. Após tais tentativas de contato, a coleta de dados se encerrou.

Os dados referentes à caracterização das disciplinas (especificas de saúde do idoso e não especificas) foram apresentados em frequência absoluta e relativa. Já as ementas deram origem a um corpus que foi analisado pelo Software Interface de $\mathrm{R}$ pourles Analyses Multidimensionnelles de Textes et de Questionnaires (IRaMuTeQ $\left.{ }^{\circledR}\right)$.

Os recursos utilizados no software foram a nuvem de palavras e a análise de similitude.(10) A primeira organiza de modo gráfico as palavras em função 
da frequência que aparecem nos discursos, enquanto a segunda, baseia-se na teoria dos grafos e tem por finalidade identificar a conexão entre grupos de palavras.

O projeto de pesquisa deste estudo foi encaminhado ao Comitê Permanente de Ética em Pesquisas com Seres Humanos (COPEP) da Universidade Estadual de Maringá e, por utilizar dados de domínio público, foi dispensado da apreciação ética.

\section{RESULTADOS}

Foram identificados no sistema e-MEC 60 IES no estado do Paraná, públicas e privadas, que disponibilizavam cursos de graduação em enfermagem ativos. Das IES, 38 disponibilizavam na página oficial online os dados referentes à grade curricular, 13 sobre grade curricular e ementa das disciplinas e nove não disponibilizavam ambos.

Com os dados obtidos dos sites oficiais, somados aos solicitados via e-mail e telefone obteve-se informações de 52 cursos de graduação. Destas, 32 disponibilizaram apenas a grade e 20 a grade e ementa.

Não se obteve nenhuma informação de oito IES. Quanto à caracterização da disciplina, notou-se que pouco mais da metade dos cursos $(53,9 \% \mathrm{n}=28)$ ofertam disciplinas específicas de saúde do idoso. Os demais cursos $(46,1 \% \mathrm{n}=24)$ abordam o cuidado à pessoa idosa em disciplinas mistas, isto é, disciplinas que abordam outras temáticas, não sendo direcionadas apenas à enfermagem gerontológica.

A nuvem de palavras, representada pela figura 1 , foi gerada a partir da análise do corpus das 20 ementas disponíveis. Nesta, os termos mais citados foram: idoso $(n=35)$; enfermagem $(n=$ $23)$; saúde $(n=21)$ e; adulto $(n=19)$.

A análise de similitude permitiu a visualização da conexão entre as palavras contidas nas ementas e como estavam correlacionadas na descrição dos
Figura 1. Nuvem de palavras: o cuidado ao idoso nos cursos de graduação em enfermagem do Paraná.

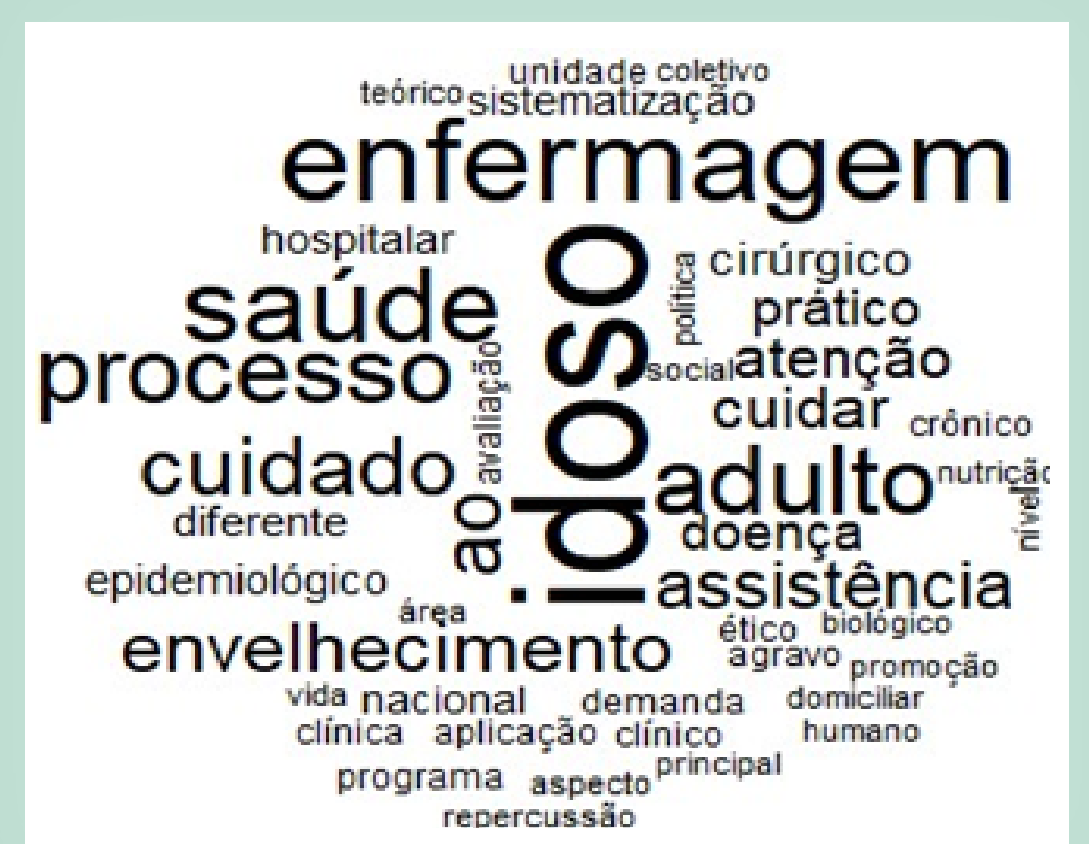

Fonte: Elaborada pelos autores por meio do software IraMuTeQ®

Figura 2. Análise de similitude: o cuidado ao idoso nos cursos de graduação em enfermagem do Paraná.

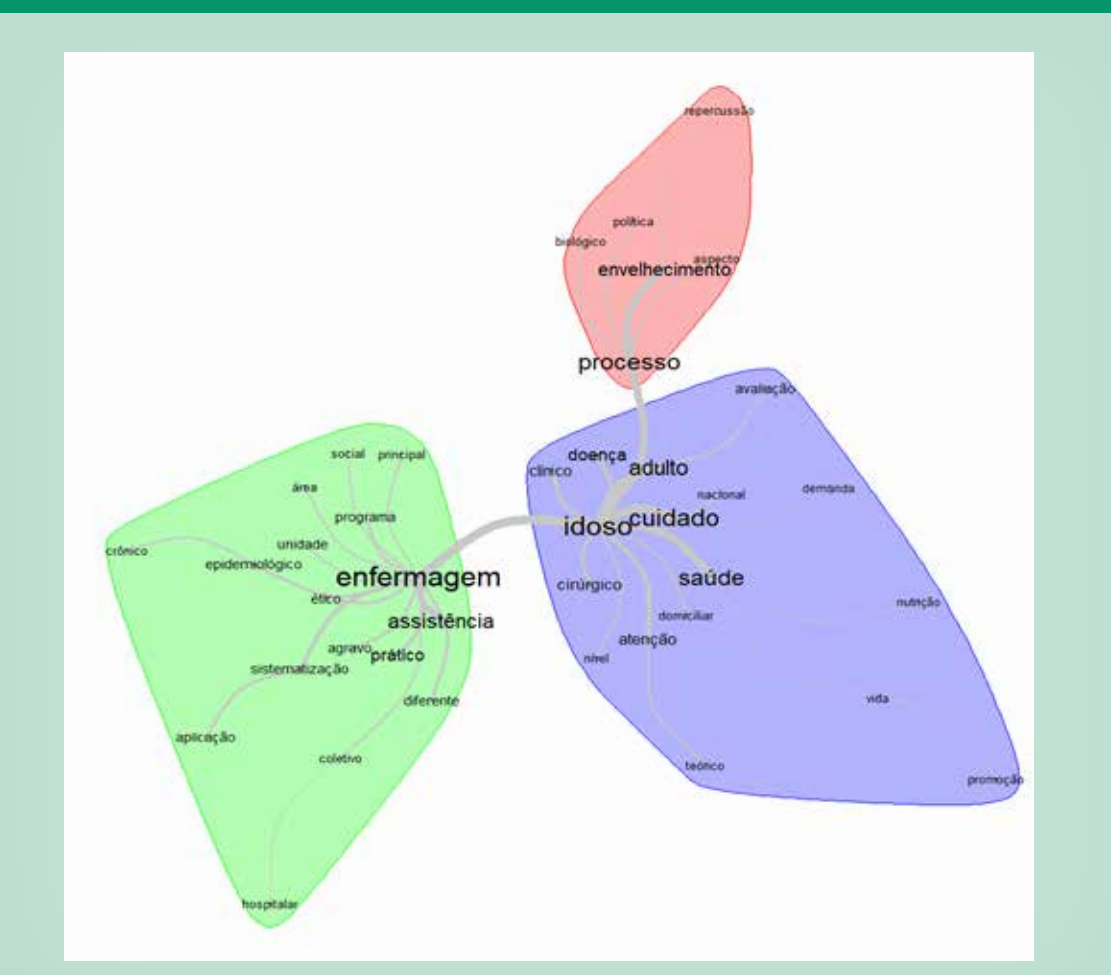

Fonte: Elaborada pelos autores por meio do software IraMuTeQ® 
conteúdos voltados ao cuidado da pessoa idosa, conforme consta na figura 2. Os eixos organizadores encontrados foram: idoso, enfermagem e processo.

\section{DISCUSSÃO}

A abordagem ao idoso foi identificada em todos os cursos analisados, associada com outra área, como a saúde do adulto, ou de forma específica, na saúde do idoso. Pouco mais da metade $(53,9 \%)$ das IES paranaenses abordam o cuidado ao idoso em disciplinas específicas, o que difere do panorama nacional, em que $34,4 \%$ das IES ofertam com essa estrutura curricular.(11)

É essencial que, cada vez mais, o conteúdo relacionado à saúde do idoso esteja inserido nas grades curriculares das IES, especialmente em disciplinas específicas. Pondera-se que dessa forma, o ensino consiga abranger de forma mais adequada, os aspectos da multidimensionalidade da saúde do idoso e, também, da heterogeneidade, do qual é cercado o processo de enveIhecimento.

Sobre o recurso de análise nuvem de palavras, notou-se que as palavras em destaque enaltecem a responsabilidade do enfermeiro como protagonista no processo de cuidado a pessoa idosa. Entende-se, pela frequência das palavras, que o ensino da saúde do idoso propõe-se a abordar os aspectos relacionados ao perfil do envelhecimento, as demandas sociais e de saúde advindas desse processo e os aspectos inerentes ao cuidado das pessoas com 60 anos ou mais. A palavra 'adulto' foi a quarta mais frequente, possivelmente porque a saúde do idoso é abordada em conjunto com saúde do adulto em parte das IES e a análise das ementas englobou-as integralmente.

A análise de similitude trouxe indicações sobre a conexidade entre as palavras. Na classificação hierárquica possuem maior representatividade as palavras 'idoso' e 'enfermagem' e 'pro-
Ressalta-se que a formação do enfermeiro deve abranger o desenvolvimento de competências para - cuidado à pessoa idosa, alinhada a políticas públicas da área e também ao modelo de atenção vigente. No entanto, pesquisa realizada com enfermeiros da Atenção Primária em Saúde (APS) evidenciou que tais profissionais não

se sentem aptos para atenderem a população idosa no que se refere à realização da avaliação multidimensional cesso' que se conectam com outras. $\mathrm{O}$ eixo 'idoso' coloca que o idoso é objeto de atenção e cuidado; o eixo processo trata do processo de envelhecimento e suas repercussões e; o eixo 'enfermagem', conecta-se com palavras que denotam existência de conteúdo sobre a sistematização da assistência nos diversos campos de atuação do enfermeiro.

É importante ponderar que o conceito de saúde no envelhecimento se traduz para além da ausência de doença, ao considerar, sobretudo, a capacidade funcional, ou seja, a autonomia (capacidade de decisão) e independência (capacidade de execução) dos indivíduos para realização de atividades que são importantes para si .(12) Nesse sentido, chamou atenção, ao analisar as ementas a ausência de termos que remetam à funcionalidade do indivíduo idoso.

Ressalta-se que a formação do enfermeiro deve abranger o desenvolvimento de competências para o cuidado à pessoa idosa, alinhada a políticas públicas da área e também ao modelo de atenção vigente.(11) No entanto, pesquisa realizada com enfermeiros da Atenção Primária em Saúde (APS) evidenciou que tais profissionais não se sentem aptos para atenderem a população idosa no que se refere à realização da avaliação multidimensional. Tal dificuldade esteve relacionada à lacuna na formação acadêmica quanto às competências para o atendimento do público idoso, as quais foram obtidas a partir de experiências na prática profissional .(13)

Um aspecto que deve ser contemplado na formação do enfermeiro relaciona-se ao entendimento do modelo de atenção utilizado para organizar o sistema de saúde. Do mesmo modo, é necessário compreender os arranjos da Rede de Atenção à Saúde RAS e a competência de cada nível. É importante dar visibilidade a APS, que deve conhecer os idosos da sua área de atuação, utilizando-se da territorialização, 
pontuando os fatores que interferem no processo saúde-doença e estratificando os idosos quanto à sua vulnerabilidade. Isto a fim de direcionar e dimensionar o atendimento em todos os níveis de atenção.(12)

Para além da análise da inserção do conteúdo relacionado à saúde do idoso nas grades curriculares das IES é necessário refletir sobre as estratégias de ensino-aprendizado utilizadas. Estudo de revisão integrativa, cujo objetivo foi analisar a formação de estudantes de enfermagem no cuidado ao idoso, evidenciou o uso predominante da metodologia tradicional, com foco na transmissão do conhecimento .(14)

$\mathrm{O}$ uso de metodologias ativas pode favorecer o processo de ensino-aprendizagem e, por conseguinte, a formação de habilidades e competências dos estudantes tornando-os autônomos no futuro cenário de prática profissional, capazes de desenvolverem novas maneiras de solucionar problemas frente às necessidades biopsicossociais dos idosos.(15)

Revisão integrativa apontou que estudantes de enfermagem apresentam uma atitude negativa em relação ao en- gajamento na atuação em gerontologia. (16) Outros estudos demonstraram, sob a perspectiva de alunos, que professores não destacam o potencial de avanço nas carreiras em gerontologia(17) e que a habilidade dos professores de enfermagem gerontológica e a disposição dos alunos para atuar na área estão associadas. Isto apresenta um desafio ao corpo docente, que deve contar com quadro de pessoal com competências para desenvolver e manter uma atitude positiva em relação à atuação em gerontologia entre os estudantes.(18)

Há relevância também os cenários de prática que se configuram como ferramentas articuladoras para o desenvolvimento das competências. Estes podem ser adotados ao considerar os setores e pontos de atenção que prestam assistência aos idosos. No setor saúde, os cenários de prática podem ser o domicílio do idoso, as Unidades Básicas de Saúde, Atenção Ambulatorial Especializada e a Atenção Terciária (hospitais). Já na assistência social, os centros de convivência, centro dia e Instituições de Longa Permanência se configuram como alternativas.

Quanto à limitação do estu- do, destaca-se a dificuldade de acesso às informações nas páginas oficiais das IES, a indisponibilidade das ementas online e, posteriormente, a falta de respostas de algumas instituições. Estes aspectos dificultaram a expressão do panorama da oferta das disciplinas nas IES paranaenses.

\section{CONCLUSÃO}

Pouco mais da metade dos cursos de enfermagem do estado Paraná aborda o cuidado ao idoso em disciplinas específicas. A análise das ementas permitiu entender que o ensino do cuidado ao idoso se propõe a abordar o processo de envelhecimento populacional e suas repercussões. Nestas, entende-se também, que o idoso é objeto de cuidado e atenção, o qual deve ser realizado de maneira sistematizada, nos diversos níveis e pontos de atenção.

Pondera-se importante que as IES avaliem se a proposta das ementas e as metodologias de ensino-aprendizagem utilizadas instrumentalizam, de fato, os futuros enfermeiros com competências para o cuidado à pessoa idosa nos diversos níveis e área de atuação.

\section{Referências}

1. Travassos GF, Coelho AB, Kuenning MPA. The elderly in Brazil: demographic transition, profile, and socioeconomic condition [Internet]. 2020 [cited 2021 Nov 02] Revista Brasileira de Estudos de População, v37, 1-27, e0129. Available from: https://doi.org/10.20947/S0102-3098a0129

2. Nascimento MM. Healthy aging in Brazil: odyssey or strategy? [Internet]. 2021 [cited 2021 Set 03] Education Gerontology, 47:9, 419-431. Available from: https://doi.org/10.1080/03601277.2021.1988209

3. Brasil. Lei $n^{\circ} 2.528$, de 19 de outubro de 2006. Aprova a Política Nacional de Saúde da Pessoa Idosa. Brasília, DF, 19out. 2006.

4. Brasil. Lei $n^{\circ} 8.842$, de 4 de janeiro de 1994. Aprova a Política Nacional do Idoso. Brasília. DF, 4 jan. 1994.

5. Brasil. Lei $n^{0} 10.741$, de $1^{\circ}$ de outubro de 2003. Estatuto do idoso. Brasília. DF, 1 out. 2003.

6. Brasil. Resolução $n^{\circ}$ 4, Conselho Nacional de Educação/Câmara de Educação Superior. Institui diretrizes curriculares nacionais do curso de graduação da área de saúde. Ministério da Educação. Diário Oficial da União. Brasília, 9 nov. 2001.
7. Tohmola A, Saarnio R, Mikkonen K, Kyngs H, Elo S. Competencies relevant for gerontological nursing: Focus-group interviews with professionals in the nursing of older people [Internet]. 2021 [cited 2021 Set 25] Nordic Journal of Nursing Research. Avalilabe from: https://doi. org/10.1177/20571585211030421

8. Silva CJA, Cassiano AN, De Lima MCRAA, Peruhype RC, De Queiroz AAR, De Menezes RMP. Perspectivas da Prática Avançada de Enfermagem no processo de cuidado gerontológico: revisão integrativa. Rev. Eletr. Enferm.

[Internet]. 2021 [cited 2021 Set 05] 23:68003, 1-12. Available from: https://www.revistas.ufg.br/fen/article/view/68003/37305

9. Chrizostimo MM, Caldas CP. Formação profissional de enfermeiro gerontológico: revisão integrativa. Research, Society and Development [Internet]. 2021 [cited 2021 Set 22] v. 10, n.11, e426101119813. ISSN 2525-3409 Available from: http://dx.doi.org/10.33448/rsd-v10i11.19813

10. Acauan LV, Abrantes CV, Stipp MAC, Trotte LAC, Paes GO, Queiroz ABA. Utilização do software iramuteq para análise de dados qualitativo na enfermagem: um ensaio reflexivo [Internet]. 2020 [cited 2021 Nov 12] 
Nursing Journal of Minas Gerais v.24:e1326 ISSN: 2316-9389. Available from: http://www.dx.doi.org/10.5935/1415-2762.20200063

11. Rodrigues RAP, Bueno AA, Silva LM, Kusumota L, Almeida VC, Giacomini SBL, et al. 0 ensino de enfermagem gerontológica nas instituições públicas brasileiras de ensino superior [Internet]. 2018 [cited 2021 Nov 02] Acta Paulista de Enfermagem, [s.I.],v. 31,n.3,p.313-320. Available from: https://doi.org/10.1590/1982-0194201800044

12. Brasil. Orientações técnicas para a Implementação de Linha de Cuidado para Atenção Integral à Saúde da Pessoa Idosa no Sistema Único de Saúde [Internet] Brasília, DF: 2018 [cited 2021 Nov 10]. 89p. Available from: https://repositorio.observatoriodocuidado.org/bitstream/handle/ handle/2556/linha_cuidado_atencao_pessoa_idosa.pdf?sequence $=1$ \&isAllowed=y

13. Marques GCS, Rodrigues JS, Rodrigues SG, Souza MR, Barros OS, Borges CJ. Profissional Enfermeiro: Competências e habilidades para a avaliação multidimensional da pessoa idosa. Revista Kairós-Gerontologia [Internet]. 2018 [cited 2021 Set 05] 21(2), 307-326. Available from: https://revistas.pucsp.br/kairos/article/view/40938 doi: https://doi.org/10.23925/2176-901X.2018v21i2p307-326

14. Perez CFA, Tourinho FSV, Junior PMC. Competências no Processo de Formação do Enfermeiro para o Cuidado ao Envelhecimento: Revisão Integrativa. Texto Contexto Enfermagem [Internet].2016 [cited
2021 Mai 22]; 25(4):e0300015. Available from: https://www.scielo.br/j/ tce/a/K47bsfz5XXHtKy5qNLnmDVj/abstract/?lang=pt doi: https://doi. org/10.1590/0104-07072016000300015

15. Souza EFD, Silva AG, Silva AILF. Metodologias ativas na graduação em enfermagem: Um enfoque na atenção ao idoso. Revista Brasileira de Enfermagem. Brasília [Internet] 2018 [cited 2021 Mai 08] v. 71, p. 920924. Available from: https://www.scielo.br/j/reben/a/T3MbRzVD93QZhZ7WRRDwTQQ/?lang=pt doi: https://doi.org/10.1590/0034-7167-20170150

16. Dai F, Liu Y, Ju M, Yang Y. Nursing students' willingness to work in geriatric care: An integrative review [Internet]. 2021 [cited 2021 Nov 24] Nursing Open v.8,Issue 5, p.2061-2077. Available from: https://doi. org/10.1002/nop2.726

17. Garbrah W, Kankkunen P, Valimaki T. Gerontological nurse teachers' and influence on students' willingness in older people nursing: A cross-sectional, correlational survey. Science Direct [Internet]. 2020 [cited 2021 Set 08] Available from: https://www.sciencedirect.com/science/article/pii/ S0260691719318076 doi: https://doi.org/10.1016/j.nedt.2020.104461

18. Garbrah W, Valimaki T, Kankkunen P. Facilitanting students' in older people nursing: Gerontological nurse teaches under scrutiny [Internet]. 2021 [cited 2021 Set 18] Elsevier. v.50, 102929. Available from: https://doi. org/10.1016/j.nepr.2020.102929 